\title{
Time Dependence of Step Fluctuations on Vicinal Cu(1 1 19) Surfaces Investigated by Tunneling Microscopy
}

\author{
M. Giesen-Seibert, R. Jentjens, M. Poensgen, and H. Ibach \\ Institut für Grenzfächenforschung und Vakuumphysik, Forschungszentrum Jülich, D 52425 Jülich, Germany
} (Received 9 August 1993)

Equilibrium fluctuations of steps on vicinal $\mathrm{Cu}\left(\begin{array}{lll}1 & 1 & 19\end{array}\right)$ surfaces show a power law time dependence. The exponent of $\frac{1}{4}$ provides evidence that the step edges and the terraces do not exchange atoms in the investigated temperature range. The activation energy for the emission of adatoms from kink sites on to the step edges is higher than what is expected based on recent total energy calculations.

PACS numbers: $68.35 . \mathrm{Md}, 68.35 . \mathrm{Bs}$

Scanning tunneling microscopy (STM) has developed into an efficient instrument capable of analyzing microscopic processes in quantitative manner. Several earlier investigations have focused on the structure of monatomic steps on single crystal surfaces [1-5], in an effort to determine the concentration of kinks in thermal equilibrium and the interactions between steps. At finite temperature, steps fluctuate in time by mass transport along the step edges as well as by mass exchange with the terraces and neighboring steps. Strong fluctuations in time have been observed even at room temperature for copper and silver surfaces $[1,2]$. As a consequence the position of a step on the surface can move in the time interval between two scan lines which gives rise to a "frizzy" appearance of steps. Fluctuations in the position of steps on a coarser scale have also been observed on silicon surfaces at high temperatures [6]. In this Letter we report on a quantitative study of the time dependence of step fluctuations on the $\mathrm{Cu}(1119)$ surface as a function of temperature. The mean square value of the step position is found to be proportional to $t^{1 / 4}$. By comparison to the statistical theories of one-dimensional systems and to Monte Carlo (MC) simulations it is shown that this power law results from mass transport solely alongside the steps. The activation energy for the mass transport is determined to be $E_{a}$ $=10300 \mathrm{~K}$.

Our STM of the Besocke type [7] is part of a standard UHV chamber with a base pressure of less than $10^{-10}$ Torr. The $\mathrm{Cu}(11119)$ vicinal surface consists of (001) terraces and (111) oriented monatomic steps along the [110] direction, spaced by an average distance of $24.2 \AA$. The single crystal was cut by spark erosion and polished to the desired orientation to within $0.2^{\circ}$. In order to decrease the sulfur content, the crystal was heated in 1 bar of hydrogen and argon $(1: 25)$ at $800^{\circ} \mathrm{C}$ for several hours, prior to mounting the sample in the UHV chamber. The sample was finally cleaned in UHV by about 50 cycles of sputtering and annealing. The temperature during the measurements was adjusted by radiative cooling and heating.

Figure 1 shows two conventional gray scale images of the $\mathrm{Cu}(1119)$ surface at 290 and $362 \mathrm{~K}$, respectively. The steps run from top to bottom and the surface height decreases from left to right. Since the STM provides no instantaneous image of the surface, consecutive scan lines represent spatial fluctuations of the steps as well as movement in time. Because of the step motion, the tip finds the step edge in different positions in each scan line which causes the "frizzy" [2] appearance of the steps at higher temperature [Fig. 1(b)]. At $290 \mathrm{~K}$ the time fluctuations are significantly reduced and one can also identify static (i.e., real) kinks in the tunneling image [Fig. 1(a)]. Rather than by counting the individual kinks in such images one may determine their concentration using the step correlation function $F(y)$ defined as

$$
F(y)=\left\langle\left[x(y)-x\left(y_{0}\right)\right]^{2}\right\rangle,
$$

where $x$ and $y$ are the coordinates perpendicular and along the ideal direction of the step edges. It is convenient to measure $x$ and $y$ in units of atom diameters. At sufficiently low temperatures where the spatial step fluctuations predominate in an STM image, $F(y)$ is a linear function of $y$ :

$$
F(y)=b^{2} y
$$

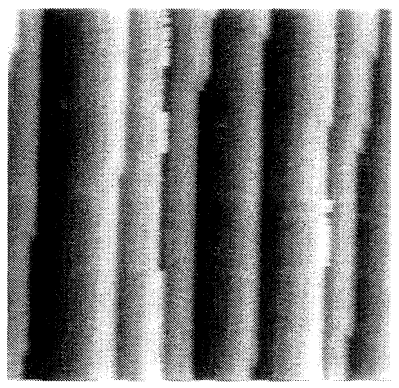

(a)

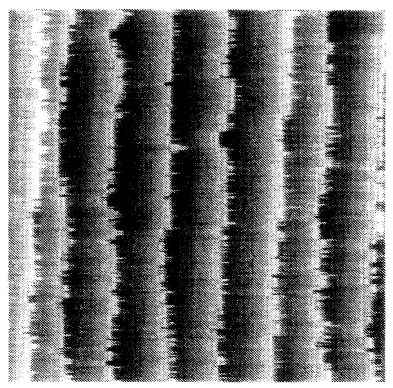

(b)
FIG. 1. Conventional $190 \times 190 \AA$ gray scale STM images of $\mathrm{Cu}(1119)$ surfaces at (a) $290 \mathrm{~K}$ and (b) $362 \mathrm{~K}$. At $290 \mathrm{~K}$ some of the apparent kinks in the image are static kinks which have not moved while they were imaged. Such kinks identify themselves by their round shape because of the finite resolution of the tip. The other kinklike structures where the position of the step changes abruptly from one scan line to the next are caused by the motion of kinks. At $362 \mathrm{~K}$ all discernible structures are caused by kink motion. 
with $b^{2}$ the mean square length of the kinks, also called the "diffusivity" [3]. $b^{2}$ is equal to the kink concentration $P_{k}$, if kinks of unit length prevail, from which the energy for the creation of a kink $E_{k}$ is determined using [1]:

$$
P_{k}=2 \exp \left(-E_{k} / k_{B} T\right) \text {. }
$$

We have determined the kink concentration at $T=290$ $\mathrm{K}$ on the $\mathrm{Cu}\left(\begin{array}{lll}1 & 1 & 19\end{array}\right)$ surface by evaluating the correlation function for a total step length of $1.5 \mu \mathrm{m}$. The result is $P_{k}=0.0119 \pm 0.0014$. The energy for the creation of a single kink is therefore

$$
E_{k}=1486 \pm 40 \mathrm{~K} \text {. }
$$

This value for the kink energy is significantly larger than the one obtained in an earlier investigation [1]. The difference is essentially due to the neglect of the time structure of tunneling images at higher temperatures in [1], as we shall see shortly. At temperatures above room temperature the step correlation function is no longer a linear function of the coordinate $y$ since the motion of kinks becomes an important factor. In order to separate the influence of the spatial fluctuations from the fluctuations in time, we applied the technique of generating time images. Here, the tunneling tip repeatedly scans the same line oriented perpendicularly to the nominal direction of the step edges, and the result is displayed as an image in which the $y$ coordinate represents the repeated scans. Hence the $y$ coordinate is equivalent to the time $t$. A step correlation function pertinent to this type of images is

$$
F(t)=\left\langle\left[x\left(y_{0}, t\right)-x\left(y_{0}, t_{0}\right)\right]^{2}\right\rangle .
$$

We have evaluated $F(t)$ from time images and $F(y)$ from conventional images for the $\mathrm{Cu}(1119)$ surface held at $362 \mathrm{~K}$ by using twenty images each and a total length of steps of $1.8 \mu \mathrm{m}$. The result is plotted in Fig. 2 as cir-

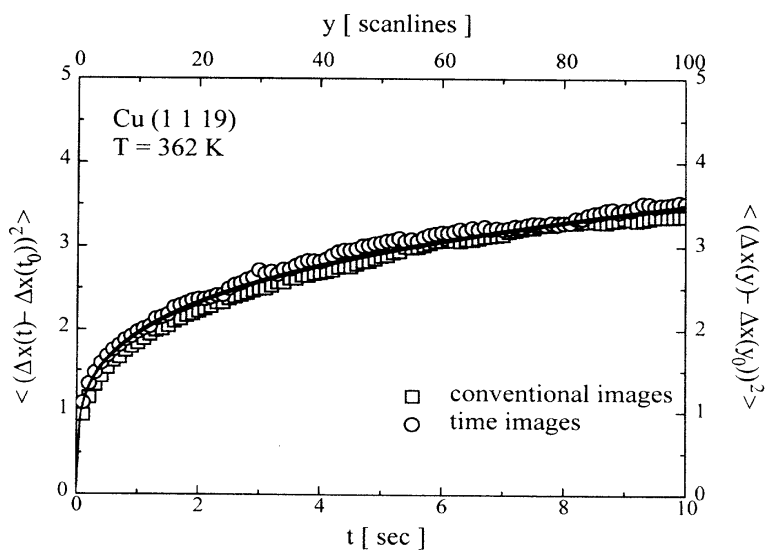

FIG. 2. The mean square displacements $F(y)$ and $F(t)$ of step positions in space images and time images taken at $362 \mathrm{~K}$ denoted by squares and circles, respectively. The full line represents a fit to a $t^{1 / 4}$ law. cles and squares, respectively, after converting the spatial coordinate $y$ into a time coordinate $t$, using the scan times of the conventional images. As can be seen from Fig. 2, there is no difference in the data outside the statistical noise which means that at $362 \mathrm{~K}$ the fluctuations of the step positions in conventional tunneling images are entirely due to the time dependence. The temperature where the influence of the time structure takes over, is at about $320 \mathrm{~K}$. The experimental data in Fig. 2 can be fitted by a $t^{1 / 4}$ power law shown as a full line in Fig. 2:

$$
F(t)=c(T) t^{1 / 4}
$$

Hence, no linear dependence, remnant of the spatial correlation [Eq. (2)] is left at $362 \mathrm{~K}$. We note in passing that below the step roughening temperature, $F(t)$ is limited by the topological constraint that steps cannot cross. Thus the $t^{1 / 4}$ law holds only as long as $F(t)$ is small compared to the square of the mean terrace width.

We have determined the temperature dependent factor $c(T)$ for a number of temperatures and the result is plotted in Fig. 3 in an Arrhenius plot. The data fit a straight line with an activation energy of

$$
E=3702 \pm 310 \mathrm{~K} \text {. }
$$

Data obtained from time images and from conventional images agree with the statistical noise, if the time scale involved in conventional images is taken into account (Fig. 3). As the temperature is reduced below $320 \mathrm{~K}$, the correlation function $F(y)$ in conventional images assumes a structure which can be fitted by a combined linear and $y^{! / 4}$ relation and the prefactor $c(T)$ was obtained from such fits.

In a previous analysis [1] of the correlation function of

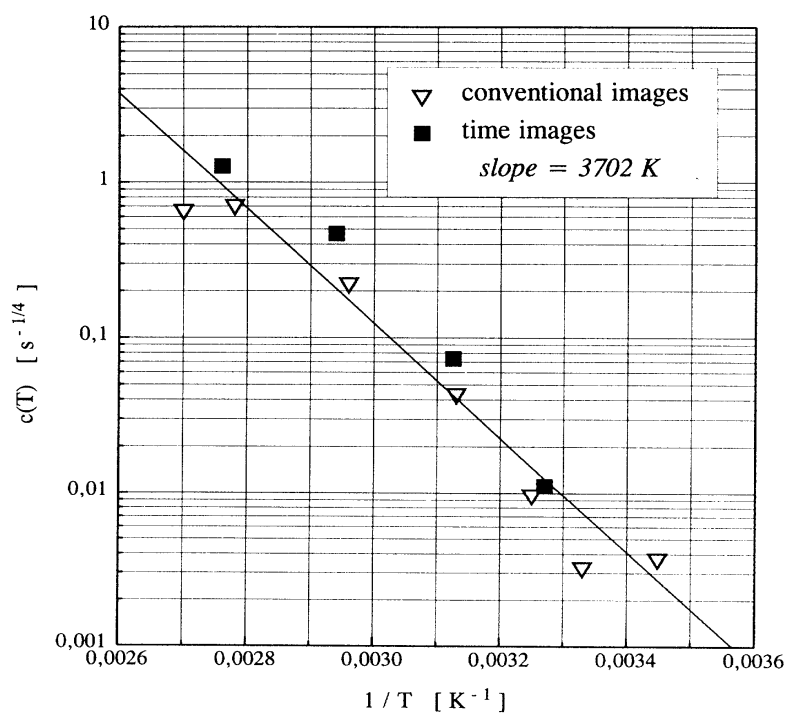

FIG. 3. Arrhenius plot of $c(T)$ defined in Eq. (6). The triangles and squares refer to data obtained from conventional and time images, respectively. 
tunneling images at room temperature, the data were fitted using the relation

$$
F(y)=a_{0}+b^{2} y .
$$

Whereas the quality of such a fit appeared quite acceptable for images taken at room temperature, the fitting constant $a_{0}$ should have been dependent on the scan time. However, the very weak time dependence is noticed only in statistical averages over a large number of data. The time dependence was therefore not detected in the earlier investigation in which only a limited data set at room temperature was available. Fitting time dependent images using the spatial correlation function (8) also resulted in too large values for $b^{2}$ in [1].

In order to explain the time and temperature dependence of the step fluctuations, one can make contact with the Langevin description of the fluctuations in linear systems [8]. For the specific case of steps, the fluctuations were calculated by Bartelt et al. [9]. Two distinctly different cases may be considered. One is where the step exchanges atoms with the terraces. In this case one has a $t^{1 / 2}$ power law for $F(t)$. In the second case, the so-called model $B$ in dynamic critical phenomena, it is assumed that mass transport occurs only alongside the step. This process is expected to prevail at lower temperatures when the kinks first become mobile. Now, the correlation function $F(t)$ is proportional to $t^{1 / 4}$. The smaller dependence on time is caused by the fact that the conservation of the number of atoms on the higher terrace introduces a correlation in the possible sequences of kink passing through a particular scan line, insofar as longer sequences of kinks of one sign become less likely. For the latter model the calculated result for $c(T)$ is [9]

$$
c(T)=0.463\left(b^{2}\right)^{3 / 4} \Gamma_{h}^{1 / 4},
$$

where $b^{2}$ is the diffusivity as defined before and

$$
\Gamma_{h}=\Gamma_{0} \exp \left(-E_{a} / k_{B} T\right)
$$

is the rate by which a kink advances by one atomic unit. Using the activation energy for $b^{2}$ one obtains

$$
E_{a}=10300 \pm 1630 \mathrm{~K} \text { and } \Gamma_{0}=10^{17} \pm{ }^{2} \mathrm{~s}^{-1}
$$

for $E_{a}$ and $\Gamma_{0}$, respectively.

The Langevin formalism does not make specific reference to the microscopic processes by which the kinks advance. In order to elucidate these processes further, we have performed MC simulations using the standard Metropolis algorithm in the canonical ensemble. Details of these calculations will be reported elsewhere together with further details of the experimental data which include other vicinal surfaces [10]. In these simulations we have assumed that the kinks advance most frequently, and therefore most effectively by the emission of one atom at a time onto the step edge. The assumption is corroborated by the relatively large preexponential factor which seems to exclude a concerted motion involving many atoms. Once the atom is emitted from a kink site, it may be either captured by the same or another kink, or it may combine with further adatoms to form a new onedimensional island, and thereby also a new pair of kinks. In order to fulfill the requirement of detailed balance, the creation of a pair of kinks by emission of an adatom from the straight step edge must also be considered as a possible process. Based on these assumptions and nearest neighbor interactions, we were able to recover Eq. (9) and the $t^{1 / 4}$ time dependence in the limit of high temperatures. Then the activation energy $E_{a}$ of $\Gamma_{h}$ is to be identified with the activation energy for the emission of an adatom from a kink (onto the step edge). This is provided that the activation barriers for an adatom to hop into a neighboring step site and into a kink site are the same. According to the total energy calculations of Tian and Rahman [11], this assumption is fulfilled to good accuracy. Hence, the activation energy of $\Gamma_{h}$ obtained from our results $\left(E_{a}=10300 \mathrm{~K}\right)$ can be compared with the calculated activation energy for the evaporation of an adatom onto a step site which was found to be $6150 \mathrm{~K}$. The experimentally determined value is, however, significantly higher than the value calculated by Tian and Rahman. In view of the good agreement between experiment and theory for the kink creation energy (1486 vs $\sim 1580 \mathrm{~K}$ [11]), a better agreement might have been expected also for the activation energies. The origin of the discrepancy is not clear at the moment. One might, however, consider the possibility that the embedded atom potential used in [11] is less accurate for surface atoms in transition states, in which case surface activation energies would also become less accurate. It should be interesting to address the issue as to how well the approximate schemes for the calculation of the total energy work also for activation energies further by comparing to other, similar methods, such as presented by the effective medium model or the glue model [12].

Helpful discussions with N. C. Bartelt, T. L. Einstein, J. Frohn, M. G. Lagally, and D. L. Mills are gratefully acknowledged.

[1] M. Poensgen, J. F. Wolf, J. Frohn, M. Giesen, and H. Ibach, Surf. Sci. 274, 430 (1992).

[2] J. F. Wolf, V. Vicenzi, and H. Ibach, Surf. Sci. 249, 233 (1991); M. Giesen, J. Frohn, M. Poensgen, J. F. Wolf, and H. Ibach, J. Vac. Sci. Technol. A 10, 2597 (1992).

[3] N. B. Bartelt, T. L. Einstein, and E. D. Williams, Surf. Sci. Lett. 240, L591 (1990); B. S. Swartzentruber, Y. W. Mo, R. Kariotis, M. G. Lagally, and M. B. Webb, Phys. Rev. Lett. 65, 1913 (1990).

[4] J. Lapujoulade, J. Perreau, and A. Kara, Surf. Sci. 129, 59 (1983); F. Fabre, B. Salanon, and J. Lapujoulade, Solid State Commun. 64, 1125 (1987); M. den Nijs and E. K. Riedel, Phys. Rev. Lett. 55, 1689 (1985).

[5] C. Jayaprakash, C. Rottman, and W. F. Saam, Phys. Rev. B 30, 6549 (1984); J. Frohn, M. Giesen, M. 
Poensgen, J. F. Wolf, and H. Ibach, Phys. Rev. Lett. 67, 3543 (1991); A. C. Redfield and A. Zangwill, Phys. Rev. B 46, 4289 (1992); S. Balibar, C. Guthmann, and E. Rolley, Surf. Sci. 283, 290 (1993).

[6] C. Alfonso, J. M. Bermond, J. C. Heyraud, and J. J. Metois, Surf. Sci. 262, 371 (1992); J. Tersoff, A. W. Denier van der Gon, and R. M. Tromp, Phys. Rev. Lett. 70, 1143 (1993).

[7] K. H. Besocke, Surf. Sci. 181, 145 (1987); J. Frohn, J. F. Wolf, K. H. Besocke, and M. Teske, Rev. Sci. Instrum. 60, 1200 (1989).
[8] P. C. Hohenberg and B. I. Halperin, Rev. Mod. Phys. 49, 435 (1977).

[9] N. C. Bartelt, J. L. Goldberg, T. L. Einstein, and E. D. Williams, Surf. Sci. 273, 252 (1992).

[10] M. Giesen-Seibert, R. Jentjens, M. Poensgen, and H. Ibach (to be published).

[11] Z.-J. Tian and T. S. Rahman, Phys. Rev. B 47, 9751 (1993).

[12] K. W. Jacobson, J. K. Nørskov, and M. J. Puska, Phys. Rev. B 35, 7423 (1987); G. Mazzeo, G. Jug, A. C. Levi, and E. Tosatti, Europhys. Lett. 22, 39 (1993). 


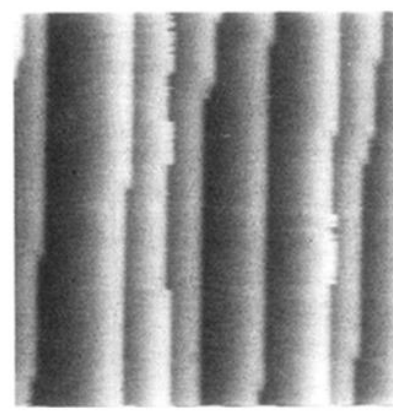

(a)

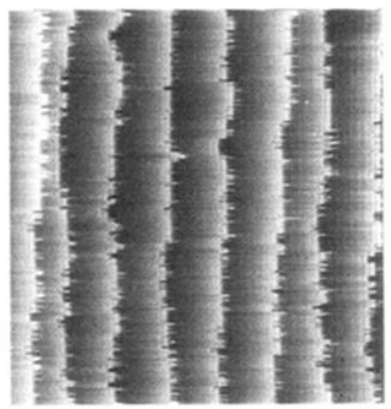

(b)

FIG. 1. Conventional $190 \times 190 \AA$ gray scale STM images of $\mathrm{Cu}(1119)$ surfaces at (a) $290 \mathrm{~K}$ and (b) $362 \mathrm{~K}$. At $290 \mathrm{~K}$ some of the apparent kinks in the image are static kinks which have not moved while they were imaged. Such kinks identify themselves by their round shape because of the finite resolution of the tip. The other kinklike structures where the position of the step changes abruptly from one scan line to the next are caused by the motion of kinks. At $362 \mathrm{~K}$ all discernible structures are caused by kink motion. 